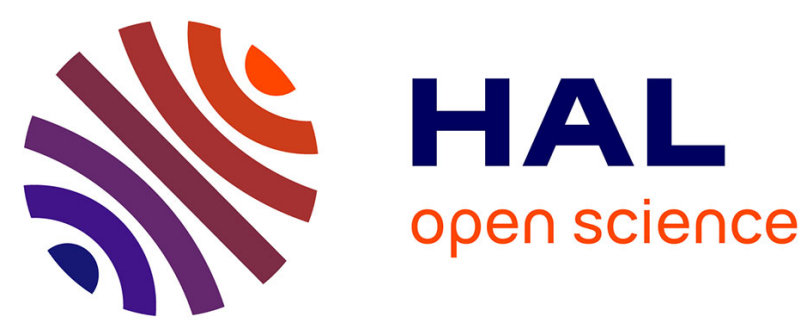

\title{
Finding the Minimum Perceivable Size of a Tactile Element on an Ultrasonic Based Haptic Tablet
}

\author{
Farzan Kalantari, Laurent Grisoni, Frédéric Giraud, Yosra Rekik
}

\section{To cite this version:}

Farzan Kalantari, Laurent Grisoni, Frédéric Giraud, Yosra Rekik. Finding the Minimum Perceivable Size of a Tactile Element on an Ultrasonic Based Haptic Tablet. ISS '16, 11th ACM International Conference on Interactive Surfaces and Spaces, Nov 2016, Niagara Falls, ON, Canada. pp.6, 10.1145/2992154.2996785. hal-01381314

\section{HAL Id: hal-01381314 \\ https://hal.science/hal-01381314}

Submitted on 14 Oct 2016

HAL is a multi-disciplinary open access archive for the deposit and dissemination of scientific research documents, whether they are published or not. The documents may come from teaching and research institutions in France or abroad, or from public or private research centers.
L'archive ouverte pluridisciplinaire HAL, est destinée au dépôt et à la diffusion de documents scientifiques de niveau recherche, publiés ou non, émanant des établissements d'enseignement et de recherche français ou étrangers, des laboratoires publics ou privés. 


\section{Finding the Minimum Perceivable Size of a Tactile Element on an Ultrasonic Based Haptic Tablet}

\author{
Farzan Kalantari \\ University of Lille 1 - Science \\ and Technology \\ CRIStAL, INRIA Lille \\ Villeneuve d'Ascq, France \\ farzan.kalantari@inria.fr \\ Laurent Grisoni \\ University of Lille 1 - Science \\ and Technology \\ CRIStAL, CNRS, INRIA Lille \\ Villeneuve d'Ascq, France \\ laurent.grisoni@univ-lille1.fr \\ Frédéric Giraud \\ University of Lille 1 - Science \\ and Technology \\ L2EP, CNRS \\ Villeneuve d'Ascq, France \\ frederic.giraud@univ-lille1.fr
}

Permission to make digital or hard copies of part or all of this work for personal or classroom use is granted without fee provided that copies are not made or distributed for profit or commercial

advantage and that copies bear this notice and the full citation on the first page. Copyrights for third-party components of this work must

be honored. For all other uses, contact the Owner/Author.

Copyright is held by the owner/author(s).

ISS '16, November 06-09, 2016, Niagara Falls, ON, Canada

ACM 978-1-4503-4248-3/16/11.

http://dx.doi.org/10.1145/2992154.2996785

\begin{abstract}
Tactile devices with ultrasonic vibrations (based on

squeeze film effect) using piezoelectric actuators are one of the existing haptic feedback technologies. In this study we have performed two psychophysical experiments on an ultrasonic haptic tablet, in order to find the minimum size of a tactile element on which all the users are able to perfectly identify different types of textures. Our results show that the spatial resolution of the tactile element on haptic touchscreen actually varies, depending on the number and types of tactile feedback information. A first experiment exhibits three different tactile textures, chosen as being easily recognized by users. We use these textures in a second experiment, and evaluate minimal spatial area on which the chosen set of textures can be recognized. Among other, we find the minimal size depends on the texture nature.
\end{abstract}

\section{Author Keywords}

Haptic; Tactile device; Friction modulation; Ultrasonic vibrations; Minimum size; Texture rendering.

\section{ACM Classification Keywords}

H.5.2 [User Interfaces]: Input devices and strategies. 


\section{Introduction}

Eye and ear are used by humans for retrieving most of information in the real, physical, world; both sense channels are quite well handled by technology. But the sense of touch in human is still complex and varies on different people and is not an absolute phenomenon. The tactile information perception might be influenced by environmental conditions such as temperature and strongly based on the skin mechanoreceptors of the human finger. Therefore the existing interactive technologies allowing to provide user to retrieve information through tactile sense are still emerging and many parameters appear to make it difficult for applications to handle tactile feedback in a shared, generic way. In particular, each tactile technology still demands a specific development and adaptation [13].

In the field of technologies for visual feedback, the concept of pixel had a huge consequence on display technology and provides a conceptual common basis between higher level software architectures, and lower-level, electronic systems that is used for display. Such common concept is still missing for tactile feedback; we still lack a common, shared reference of an elementary tactile information, both in terms of spatial resolution, and information nature; can we go up to a normalization level comparable to the one that pixels provide for visual information?

In this study we introduce a step toward the concept of taxel (Tactile Element), by suggesting an up-down response protocol (adaptive procedure) in psychophysics

[5] that allows, for a specific tactile feedback technology, to measure the minimum size on which tactile information can be retrieved. We define the notion of texture waveform as the elementary signal shape that we provide to the tactile feedback technology, that is used as periodical signal for tactile stimulation. Such notion is a standard, usable term for all existing tactile feedback technologies (see related work section). We provide a first experiment that identifies few elementary textures that can be easily identified by user. Among the 24 textures proposed to user, we identify three that all users can identify accurately. From this experiment, we derive a second experiment that evaluates the minimum size of the tactile element at which user can differentiate textures accurately. We conclude that the texture type influences the minimum size of the tactile element.

\section{Related Work}

In the recent years, touchscreen interfaces with haptic feedback to the user's finger have become more and more popular. This haptic feedback permits the users to enrich the feeling of what they touch and can improve the performances of their interactions.[3, 6, 14]

Generally, we can divide the haptic feedback technology on touchscreen interfaces in to 4 categories. These technologies mostly use various friction modulation between the fingertip and the interaction surface for texture rendering. The first class of devices are based on electro-vibration effect which use electrostatic force, generated by applying a voltage to the surface for haptic feedback $[2,8]$. The second category create haptic feedback through electroadhesion technique, which also use electrostatic force generated by DC excitation to the haptic surface. [9]. Both of these two classes of devices increase the friction between the fingertip and the interaction surface. The third class of devices are based on friction reduction using ultrasonic vibration via the squeeze film effect $[1,12,7]$. This effect reduces the friction, which is opposite to electrostatic force in the two previous technologies. The fourth class of devices utilize 
vibrotactile actuators for tactile rendering which is widely used in smartphones and tablets since several years now [4]. Furthermore, in [10] the differences in haptic perception of object size when exploring the inside or outside the objects is investigated by means of psychophysical experiments. But there is still no studies to show the minimum object size for tactile perception while using a touchscreen device with haptic feedback.

\section{Experiment 1: Finding the Three Perceivable and Distinguishable Textures}

At first we have done an experiment in order to find at least 3 different textures that could be perceived and distinguished properly by all users. We have selected 3 types of textures due to the time constraints for performing the two experiments by participants.

\section{Apparatus}

We used a haptic tablet based on ultrasonic vibrations for texture rendering [11] which is developed on a Banana $\mathrm{Pi}$, a single-board computer with $1 \mathrm{GHz}$ ARM Cortex-A7, dual-core CPU and $1 \mathrm{~GB}$ of RAM working in parallel with STM32f4 microcontroller. The communication between the singleboard computer and the microcontroller is provided via the Serial Peripheral Interface (SPI) bus at $10 \mathrm{kHz}$. This singleboard computer is connected to a 5 inches capacitive touchscreen in order to detect the finger position on the display with the sampling frequency of 50 $\mathrm{Hz}$. The two piezoceramic actuators are placed at each side of the display.

\section{Design and Procedure}

In order to design our first experiment, we have defined 4 types of texture waveforms as square, sine, dirac and sawtooth with the constant amplitude of $1 \mu \mathrm{m}$ and the spatial frequencies of $50 \mu \mathrm{m}, 100 \mu \mathrm{m}, 500 \mu \mathrm{m}, 1000 \mu \mathrm{m}$,
$5000 \mu \mathrm{m}$ and $10000 \mu \mathrm{m}$. The setup of the first experiment is illustrated in figure 1 which was coded with Java and Processing language and the display is divided to 4 equal sections. On each part there is a specific texture waveform and so the value of spatial frequency can be selected. Ten participants ( 4 female and 6 male) were free to explore the surface as long as they wanted and then choose 3 or 4 textures that could be accurately sensed and distinguished among all the 24 provided textures.

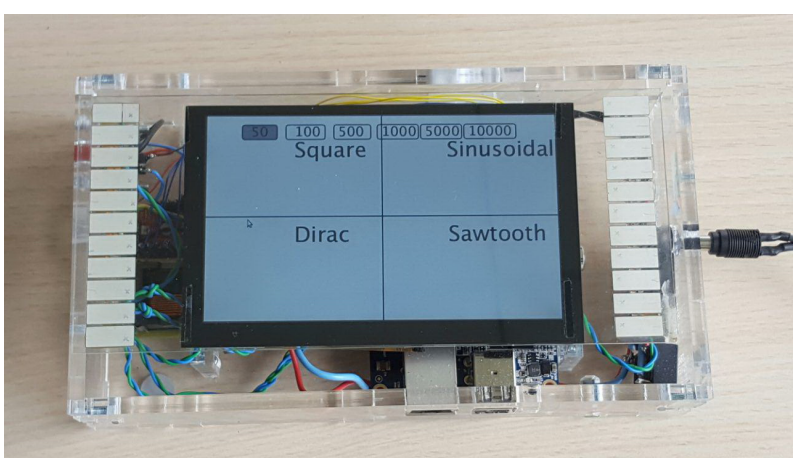

Figure 1: The setup of the Experiment 1

\section{Results}

The analysis of results as we can see in table 1 , show that several participants could distinguish between 5 to 7 different textures. In overall the results demonstrate that all $100 \%$ of participants could perceive and distinguish the following textures perfectly: square texture, sinusoidal texture and dirac texture with the spatial frequency of $50 \mu \mathrm{m}, 1000 \mu \mathrm{m}$ and $1000 \mu \mathrm{m}$ respectively and the constant amplitude of $1 \mu \mathrm{m}$ for all of the textures. The feeling perception of the dirac and sawtooth texture waveforms were more or less similar for the participants and therefore rather hard to be distinguished appropriately. These results are used for our second experiment. 


\begin{tabular}{|c||c|c|c|c|}
\hline Participants & Square $(\mu \mathrm{m})$ & Sinusoidal $(\mu \mathrm{m})$ & Dirac $(\mu \mathrm{m})$ & Sawtooth $(\mu \mathrm{m})$ \\
\hline 1 & 50 & 1000 & 1000 & 5000 \\
2 & 50 & 1000 & 1000 & 10000 \\
3 & 50 & 1000 & 1000 & 5000 \\
4 & 50 & 500,1000 & 1000 & $500,1000,5000$ \\
5 & $50,1000,5000$ & 1000 & 1000 & 5000 \\
6 & 50,1000 & 500,1000 & 1000 & 5000,10000 \\
7 & 50,1000 & 500,1000 & 500,1000 & 10000 \\
8 & 50,1000 & 1000 & 500,1000 & 10000 \\
9 & 50 & 1000 & 500,1000 & 1000 \\
10 & 50 & 1000 & 1000 & 10000 \\
\hline
\end{tabular}

Table 1: The results of the chosen textures from experiment 1

\section{Experiment 2: Finding the Minimum Size of a Tactile Element}

The goal of our second experiment is to determine the minimum size of a tactile element (taxel) on the touchscreen on which the participants are able to

distinguish the 3 textures found from the results of the experiment 1 . As before we have used the same haptic tablet based on ultrasonic vibrations and defined 3 textures due to the time constraints to accomplish the task by the participants

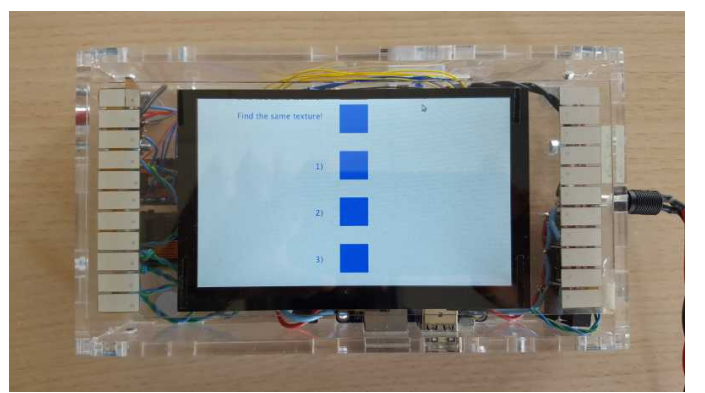

Figure 2: The setup of the minimum size experiment
The participants were demanded to find the accurate corresponding texture of the first taxel among the 3 other taxels and clicked on (1), (2) or (3) on the keyboard for the correct answer.

\section{Design and Procedure}

We have used an up-down response protocol (adaptive procedure) in psychophysics [5] with the total number of 25 tries for each of 3 trials. Thus the minimum size of the tactile elements is considered to be the last size as the ten participants obtained after 25 tests for each trial (10 participants $\times 3$ textures $\times 25$ times $=750)$. We have displayed 4 tactile elements of square shapes with the identical initial size of $10 \mathrm{~mm}(1 \mathrm{~cm})$ that are situated in parallel as shown in figure 2. At each try if the participants has a correct answer for the texture detection, the size of all squares decrease by $0.5 \mathrm{~mm}$ simultaneously and for the wrong answers it will increased by $0.5 \mathrm{~mm}$. At each step there is a specific texture on the first tactile element and the textures on the other taxels are assigned 


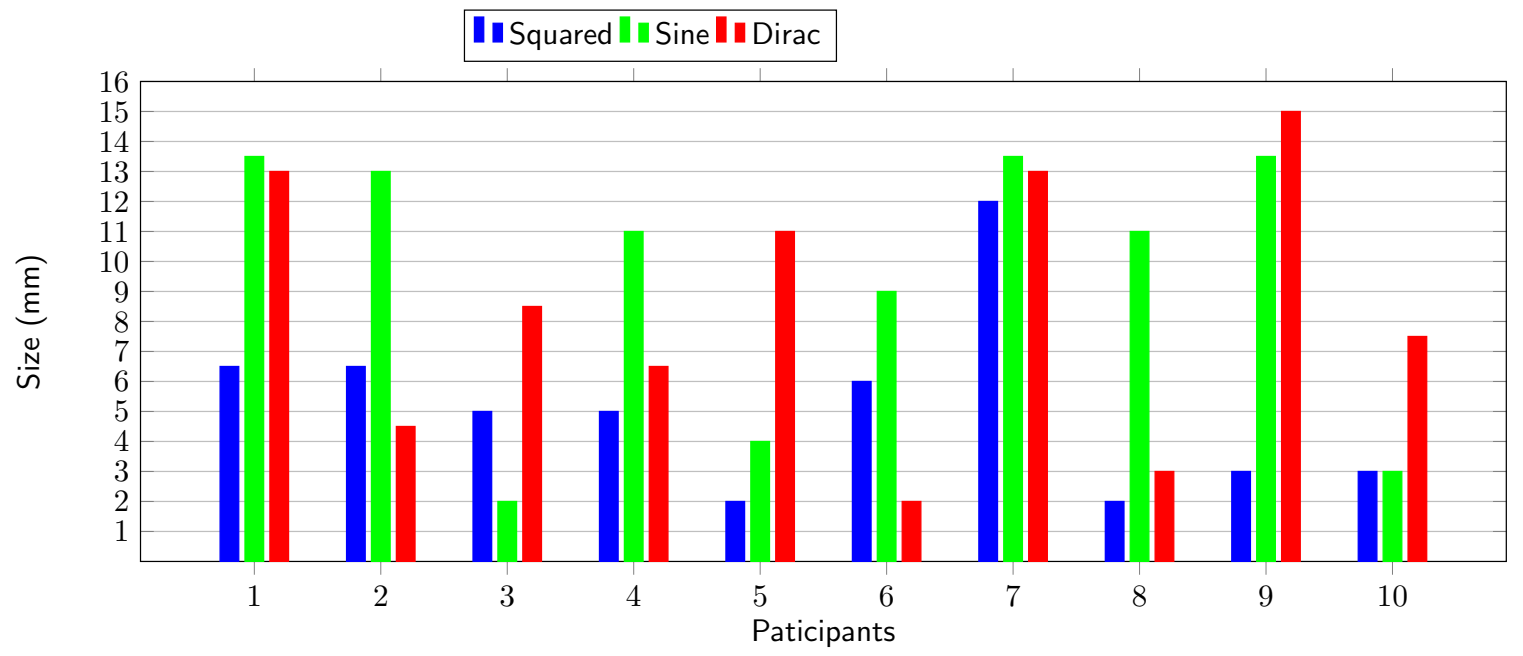

Figure 3: The results of each participant for the minimum size of the tactile element for the 3 given textures

with the randomized manner, in order to obtain the ideal validity of the experiment.

\section{Results and Discussions}

For the first trial we applied a square signal waveform (texture) with the spatial frequency of $50 \mu \mathrm{m}$ and the constant amplitude of $1 \mu \mathrm{m}$ to the first square object (taxel) and asked the participants to find it among the 3 following squares with a randomized texture on each one. As it is illustrated in figure 3 , all of 10 participants were able to distinguish the given square texture with the other textures for the minimum size of $\underline{12 \mathrm{~mm}}$. $(6.5 \mathrm{~mm}$ for 9 participant)

In the second trial we have exerted a sine wave with the spatial frequency of $1000 \mu \mathrm{m}$ and the amplitude of $1 \mu \mathrm{m}$ to the first square. The procedure is the same as the first trial. The result is illustrated in the figure 3. As we find out from figure 3 , the minimum size of the square is $13.5 \mathrm{~mm}$ for all $100 \%$ of the participants to distinguish the provided texture.

In the third trial with the same procedure as before, we applied a dirac texture with the spatial frequency of $1000 \mu \mathrm{m}$ and the constant amplitude of $1 \mu \mathrm{m}$ to the first square. The minimum size of the taxel is $15 \mathrm{~mm}$. (see figure 3)

\section{Conclusion and Future work}

Our results show that the minimum perceivable size of the tactile element depends on the nature of texture signal waveform. The standard range in our case is from few millimeters to about one centimeter, depending on the texture waveform. Our results also demonstrate that the 
textures with square signal might be more perceivable with small sizes $(6.5 \mathrm{~mm}$ for the majority of the participants). Hence, textures with square signal waveform is the most suitable texture for tactile rendering of small size of virtual objects on the ultrasonic based haptic feedback touchscreens.

As the future work we target to do the same study with the other haptic feedback technologies on touchscreens and try to compare the results. It is also possible to change the tactile element (square in our case) with other geometrical shapes.

\section{Acknowledgements}

This work is partially funded by European ERDF funds (IRCICA, CPER MAUVE, SCV) and ANR funding agency (equipex IRDIVE). The authors would like to appreciate Nicolas Bremard for his useful help and discussion for developing the experiments in our study.

\section{References}

[1] Amberg, M., Giraud, F., Semail, B., Olivo, P., Casiez, G., and Roussel, N. Stimtac: A tactile input device with programmable friction. In UIST 2011 (2011).

[2] Bau, O., Poupyrev, I., Israr, A., and Harrison, C. Teslatouch: Electrovibration for touch surfaces. In Proc. UIST 2010 (2010).

[3] Casiez, G., Roussel, N., Vanbelleghem, R., and Giraud, F. Surfpad: Riding Towards Targets on a Squeeze Film Effect. In Proc. CHI '11 (2011).

[4] Choi, S., and Kuchenbecker, K. J. Vibrotactile display: Perception, technology, and applications. Proc. IEEE 2013 Volume 101, No. 9 (2013).
[5] Leek, M. R. Adaptive procedures in psychophysical research. Perception \& Psychophysics (2001).

[6] Levesque, V., Oram, L., MacLean, K., Cockburn, A., Marchuk, N. D., Johnson, D., Colgate, J. E., and Peshkin, M. A. Enhancing physicality in touch interaction with programmable friction. In Proc. CHI 2011 (2011)

[7] Long, B., Seah, S. A., Carter, T., and Subramanian, $\mathrm{S}$. Rendering volumetric haptic shapes in mid-air using ultrasound. ACM Trans. Graph. (2014).

[8] Senseg. Inc. http://senseg.com/.

[9] Shultz, C. D., Peshkin, M. A., and Colgate, J. E. Surface haptics via electroadhesion: Expanding electrovibration with johnsen and rahbek. IEEE (2015).

[10] Tiest, W. M. B., and Hayward, V. Inside vs. outside: Haptic perception of object size. In Proc. WHC 2015, IEEE (2015)

[11] Vezzoli, E., Sednaoui, T., Amberg, M., Giraud, F., and Lemaire-Semail, B. Texture rendering strategies with a high fidelity - capacitive visual-haptic friction control device. In Haptics: Perception, Devices, Control, and Applications (2016).

[12] Winfield, L., Glassmire, J., Colgate, J. E., and Peshkin, M. T-pad: Tactile pattern display through variable friction reduction. IEEE (2007)

[13] Yi, B., Cao, X., Fjeld, M., and Zhao, S. Exploring user motivations for eyes-free interaction on mobile devices. CHI '12 (2012).

[14] Zhang, Y., and Harrison, C. Quantifying the targeting performance benefit of electrostatic haptic feedback on touchscreens. In Proc. ITS 2015 (2015). 